\title{
Prevention and Treatment of Skin Injury and Trauma in Triathlon Competition Day
}

\section{Keiji Sugiura* and Mariko Sugiura}

Department of Environmental Dermatology and Allergology, Daiichi Clinic, Japan

\begin{abstract}
There are many kinds of skin injuries and trauma in triathlon competition, because triathlon is three kinds of exercise. The number of triathletes is growing in the world since becoming Olympic game; however there is not any significant data of skin injuries in triathletes. Actually, skin injuries might be caused in more athletes; most of skin injuries are not serious and not counted. Our focus is skin injuries and trauma in triathlon competition, and it is possible to prevent or reduce most of skin injury by careful and safety actions.
\end{abstract}

Keywords: Triathlon; Skin; Injury; Trauma; Swimming; Bicycle; Running

\section{Introduction}

Triathlon is three kinds of exercise (swimming, bike (cycling) and running) by individual person at one day. Triathlon has been more popular because of becoming an Olympic sport. Almost triathletes wear tri-suits (one piece or separate) for a competition. Tri-pants are constituted swim wear with pad, in case of swimming; they wear wetsuits on tri-suits. During bikes or running, athletes wear tri-suits or bike-clothes on it.

The number of triathletes is growing in the world, especially in Asia, since the frequency of injury is increasing. Actually, skin injuries might be caused in more athletes; however most of skin injuries are not serious and not counted. There is not any significant data of skin injuries in triathletes; however triathletes should realize the risk of injury. A previously report showed that most injuries occurred during running (50\%) than cycling (43\%) and swimming (7\%) [1]. Egermann [2] reported that $51.1 \%$ of German speaking athletes in Ironman Europe 2000 suffered one or more contusion/skin-abrasions. Significant relations were found that high performance athletes suffered more contusions/abrasions [2].

Many athletes don't worry about skin injury, because most of skin injury is common and not serious. But secondary infection cause in the skin injury, serious condition may develop and be influenced to their active performance. Skin injuries and trauma can affect an athlete's performance, and athletes should know the risk factors, prevention and treatment of skin disorders. Early treatment could maintain performance state and high motivation. We show skin injuries and trauma of each exercise, and describe prevention and treatments. Our focus is skin injury in triathlon for enlighten, and it is possible to prevent or reduce most of skin injury by careful and safety actions.

\section{Injuries}

\section{Swimming}

The rate of injuries in swimming is significantly lower than the other 2 disciplines [3].

Blister from wet suite: Wetsuit friction cause blister on the skin, the high frequency is on the neck, nipples and lateral chest. Dirty sea water possesses possibility to cause secondary infection.

Jellyfish sting: Jellyfish often appear at the end of season. Long john (sleeveless) type wetsuit do not cover the arms, since some athletes are stung on their arms during swimming. Venomous jellyfish are often seen from area to country, and general of competition could cancel swimming.
Sea snake bite: Sea snakes always live in the deep, but sea snakes sometimes swim in the shallow sea. Sea snakes swimming in the shallow sea misunderstand attacking by athletes and they coil around athlete's arms with biting. Almost sea snakes are venom snakes. When athletes are bit by sea snakes, they stop swimming and must call for help. If athletes bitten by sea snakes continue to swim, venom spread through their bodies. It is very difficult for preventing sea snake biting.

Injury due to floating something: There are some floating, not only sea plant but also garbage and waste. Some garbage and waste with pointed tips can cause skin injuries. When athletes swim around the tetrapod, athletes often kick tetrapod and develop skin injuries.

Cold urticaria and peripheral circulation disturbance (frostbite, trench foot, hypothermia and cyanotic skin) by cold temperature [4]: Cold environmental factors develop skin injuries and loss vigor of exercise. Cold temperature develops frostbite and hypothermia. Trench foot is caused by swimming in cold sea. Cold exposures often develop cold urticaria, but most of athletes with cold urticaria can continue exercise.

Athletes hit each other: Some athletes push their way on the swimming course, since their bodies contact other athletes accidentally or purposely. Hitting by hands, foots or nails can cause skin injuries.

Urticaria or food dependent exercise induced anaphylaxes (FDEIA): Most of athletes start at two or three hours later of breakfast, these allergic reactions can develop at those times. FDEIA [5] is more serious life threatening condition than urticaria.

Cercariae sting: Cercariae cause dermatitis with severe itching to swimmers in the lake competition [6]

\section{Bike part}

Abrasion, erosion, ulcer and laceration: These traumatic injuries

*Corresponding author: Keiji Sugiura et al., Department of Environmental Dermatology and Allergology, Daiichi Clinic, Nittochi Nagoya Bld. 2F, 1-1 Sakae 2, Nakaku, Nagoya, 468-0008, Japan, Tel: +81-52-204-0834, E-mail: ksugiura@ daiichiclinic.jp

Received November 12, 2015; Accepted November 25, 2015; Published December 01, 2015

Citation: Sugiura K, Sugiura M (2015) Prevention and Treatment of Skin Injury and Trauma in Triathlon Competition Day. J Sports Med Doping Stud 6: 170 doi:10.4172/2161-0673.1000170

Copyright: $\odot 2015$ Sugiura K et al. This is an open-access article distributed under the terms of the Creative Commons Attribution License, which permits unrestricted use, distribution, and reproduction in any medium, provided the original author and source are credited. 
are most common in cyclists, and these sometimes are involved bike mechanical condition [7]. Many slipping or falling down accidents develop these injuries. Slipping is caused by not only raining but also going on the gravel, pavement and manholes. Falling down are caused by crashing, losing balance and impossible to turn on the pavement or manholes.

Sun burn: Triathlon is a summer sport, and sunburn is often seen in competitions. Serious sunburn is seen in the athletes with fair skin. In the case of using sun blocks, most of sunblock cosmetics are taken off by sweat, water (swimming and drinking) and sebum. It recommends using properly water-resistant sunblock for low sun exposure [8]. Sunburn often loose physical strength.

Insect bite: If the bike course is via mountain, you can see flying insect and bee. They hit face, neck, arms and legs, athletes are developed skin injuries and sting. Insect often come up to athletes for smelling especially jelly.

Pad and saddle-related skin injuries: Tri-suit (pad) and saddle rubbed against the thigh and inguinal skin, and athletes develop blister on these skin from the friction.

There are blister, abrasion chafing, perineal folliculitis and furuncles. Most of these might be caused in long distance competition or in beginners.

Contact dermatitis: Many athletes are exposed numerous allergens in daily cycling. If some athletes are exposed to some allergens for them, they developed contact dermatitis [9]. Contact or irritant dermatitis of thigh due to non-slip silicon on the bike shorts or tri-pants are often seen.

Vulvar swelling: In case of competition (intensive cycling), this symptom sometimes could be caused. This is associated with lymphatic dilatation, since it develops dermal fibroedema [10].

Urticaria or food dependent exercise induced anaphylaxes (FDEIA): Most of athletes start at two or three hours later of breakfast, most of these allergic reactions can develop during swimming or cycling. FDEIA shows often more serious life threatening condition than urticaria [5].

\section{Run part}

Blister from shoes: Previous report described that the most frequent injury is blisters, $50 \%$ of 76 road runners [11]. Many triathletes put on sox in the middle or long distance competition than short (standard) distance. If the triathlete don't put on the sox, blister often cause on foot by rubbing. When triathlete pour water over them, wetting their sox develop blister easily on foot.

Abrasion and laceration due to slipping or falling down: Exhausted athletes can't run trip over their own feet. These accidents develop erosion or ulcer on the skin due to slip. Dehydration loses the body balance, and then athletes could be staggering and falling down.

Sun burn: Sunlight at the running is strong. Sunblock cosmetics are taken off by sweat, water (sea and drinking) and sebum, since it recommends using sunblock at the starting to run. For decreasing sun exposure, water-resistant sunblock and short-sleeved tri-suits are useful. It recommends using properly water-resistant sunblock for low sun exposure [8]. Sunburn often loose physical strength.

Insect bite: Insect or bee flying strike athletes, then sting sometimes is caused. Insects, especially bee sometimes go up to foods smelling honey, then athletes surprise and slip or are stung.

Blister from measuring instrument: Measuring instruments are used on the leg, since these instruments often move and cause friction on the skin. In the run part, blister from measuring instruments are often seen.

Jogger's nipples: Not only female but also male athletes, long distance runners, sometimes develop painful erythema and erosion. This injury is caused by friction between nipples and clothes. Previous reports described that $2-16.3 \%[11,12]$ of runners on completion develop Jogger's nipples. Jogger's nipple is related with long distance running [13]

Jogger's toe: Subungual heamatoma is often caused. Black heels are caused by friction and pressure; it seems likely to nevus. This might be related with running form and tight shoes. Treatment for these is not necessary.

Sports purpura [14]: These cutaneous symptoms could occur after exercise. Most of them could be seen on the legs, and most of them are not necessary for treatment.

Urticaria or food dependent exercise induced anaphylaxes (FDEIA): If FDEIA cause in the running, probably its competition is short or less standard distance.

Contact dermatitis: Some triathletes know contact or irritant dermatitis due to non-slip silicon on the tri-pants. Kockentiet [9] reported that athlete's skin face and repeated exposure to numerous allergens and chemicals caused both allergic contact dermatitis (ACD) and irritant contact dermatitis (ICD).

\section{T1 (swim-bike)}

When all athletes run or walk by barefoot to T1, some athletes step on the foreign body and develop skin injuries on their soles. The frequency of developing skin injury is low. When athletes start to ride on bike, some athletes develop skin injuries after losing balance and falling down. The reason of losing balance is that athletes after swimming felt different body position against gravity.

\section{T2 (bike-run)}

Bikes of other athlete hit on, and then skin injury could be caused. When athletes get off bike, some athletes develop skin injuries after losing balance and falling down; because legs and thigh of some athletes are tired, they can't stand up right now. Some athletes take of their shoes on the bike, and they develop skin injuries after running to T2 on naked foot.

\section{Treatment and Prevention}

Most of skin injuries and trauma are able to prevent.

\section{Prevention}

Tri-suit: If tri-suits are not the size of athletes, they would develop skin blister due to friction by wetsuits. Tri-suits with high neck are good for prevention of blister. Recently short-sleeved tri-suits are often seen, these prevent sunburn on shoulders. Protective equipment (pad, bike shorts) are useful for prevention of saddle -related skin injury.

\section{Cardigan for triathlon, other clothes with long sleeves}

These clothes are useful for preventing sunburn against shoulders and arms. Long sleeves wear are recently noticed on age class athletes. Short-sleeved tri-suits prevent sunburn on shoulders for decreasing fatigue.

White petrolatum: Before competition, many athletes use white petrolatum for prevention of blister causing on their neck, lateral chest and thigh or inguinal skins from tri-suit or wetsuit. 
Sunblock: Water-resistant sunscreens are adequate protection from $\mathrm{UV}[8]$, and it is better to use this at TI and T2. For decreasing sun exposure, water-resistant sunblock and short-sleeved tri-suits are useful. It recommends using properly water-resistant sunblock for low sun exposure [8].

Sox: Sox are the most effective instrument for preventing blister on foots. When the athlete pour water over them, wetting their sox develop blister easily on foots. It is important not to pour water against their foot.

Gloves: Gloves for cycling are good instruments for preventing skin injuries at slipping or falling down. Beginners are often caused blister on the hands by friction of handle, it recommends some athletes with little experience using gloves during bike part.

Taping: Some athletes use tape on their neck and nipples for preventing blister from wetsuit or clothes. Taping possess possibility of causing itching or irritant dermatitis.

Incubation: Incubation cream is useful for prevention of peripheral circulation disturbance (frostbite, trench foot, hypothermia and cyanotic skin) by cold temperature.

Looking ahead: Looking ahead is an important and evasive action from other athletes, floating or waste on the course.

Good mechanical condition: Keeping good mechanical condition of bicycle [7] is an essential prevention of trauma and reducing skin injuries. Riding position and pad affect saddle -related injuries, blister, abrasion, folliculitis, furuncles, vulva trauma and subcutaneous perineal nodules.

Avoiding allergen foods before exercise: Most of athletes with FDEIA understand own allergens, it is important to avoid these allergens before at least three hours of exercise.

Keep standing position: Some athletes after swimming or bike loose body balance and developing skin trauma. For prevention of losing balance, it recommends to keep standing position with deep breathing and taking hold of something for ten seconds.

\section{Treatments}

Keeping clean at the skin developing injuries is most important, since cleansing is an efficacious first treatment against secondary infection.

External medicines: Steroid ointments are very often used for treatment of insect bite, contact dermatitis and jerry fish sting. Ointments including antibiotics are often used for skin erosion or ulcer after washing. Steroid spray is useful for sunburn because touching often cause pain and blister.

Steroid or anti-histamine tablets: Steroid medicines are often prescribed by physicians. Steroid or anti-histamine medicines are good for allergic reactions (urticaria, FDEIA), sting and sunburn.

Examination by physicians or hospitalization: Serious condition needs for treatment by physicians. Especially, in case of diffuse skin injuries, venom jerry fish sting, venom sea snake biting and FDEIA, it needs treatment immediately by physicians.

\section{Discussion}

Many physicians and triathletes are sensitive to skin damage by ultraviolet $[5,8,15]$, however there have been no significant reports about only skin injuries of triathletes before. From our experience, the most frequency of skin injury is blister on foot. The frequency of skin injuries might be related with performance level [2] age [2] and experience competition. But injury incidence was unrelated with amount, intensity and frequency of training [16]. Skin injuries caused in not only elite but also age class, the frequency of serious injuries in age class could be more than those of elite. Serious skin injuries could affect discontinues the triathlon. Dehydration, temperature and humidity controlling and anomaly electrolyte cause through the skin, since skin injuries cause easily these unusual condition. Skin is the largest human tissue, and then diffuse skin injuries are difficult for controlling temperature and dehydration and for continuing exercise.

\section{To Triathletes}

Calling for help and to decide retiring during competition are key of preventing serious condition, and these are most important for athletes. Athletes in age class should possess brave of retiring and calling for help, these are not shaming. If athletes develop serious injuries, serious condition affects other athletes and competition.

\section{Conflict of Interest}

There is not any conflict of interest.

\section{References}

1. Zwingenberger S, Valladares RD, Walther A, Beck H, Stiehler M, et al. (2014) An epidemiological investigation of training and injury patterns in triathletes. $J$ Sports Sci 32: 583-590.

2. Egermann M, Brocai D, Lill CA, Schmitt $H$ (2003) Analysis of injuries in longdistance triathletes. Int J Sports Med 24: 271-276.

3. Bales J, Bales K (2012) Swimming overuse injuries associated with triathlon training. Sports Med Arthrosc 20:196-199.

4. McMahon JA, Howe A (2012) Cold weather issues in sideline and event management. Curr Sports Med Rep 11: 135-141.

5. Adams BB (2002) Dermatologic disorders of the athlete. Sports Med 32: 309 321

6. Litschauer-Poursadrollah M, Mayer DE, Hemmer W, Jarisch R (2010) [Jellyfish and poison-producing animals that endanger swimmers]. Dtsch Med Wochenschr 135: 1073-1077.

7. Mellion MB (1991) Common cycling injuries. Management and prevention. Sports Med 11: 52-70.

8. Moehrle M (2001) Ultraviolet exposure in the Ironman triathlon. Med Sci Sports Exerc 33: 1385-1386.

9. Kockentiet B, Adams BB (2007) Contact dermatitis in athletes. J Am Acad Dermatol 56: 1048-1055.

10. Coutant-Foulc P, Lewis FM, Berville S, Janssen B, Guihard P, et al. (2014) Unilateral vulval swelling in cyclists: a report of 8 cases. J Low Genit Tract Dis 18: e84-89.

11. Orava S (1977) About the strains caused by a marathon race to fitness joggers. J Sports Med Phys Fitness 17: 49-57.

12. [No authors listed] (1978) More on jogger's ailments. N Engl J Med 298: 405 406 .

13. Purim KS, Leite N (2014) Sports-related dermatoses among road runners in Southern Brazil. An Bras Dermatol 89: 587-592.

14. Kluger N (2012) [Sports purpura]. Presse Med 41: 899-906.

15. Moehrle M (2008) Outdoor sports and skin cancer. Clin Dermatol 26:12-15.

16. Korkia PK, Tunstall-Pedoe DS, Maffulli N (1994) An epidemiological investigation of training and injury patterns in British triathletes. $\mathrm{Br} \mathrm{J}$ Sports Med 28: 191-196. 